\title{
A Survey on Antimicrobial Sensitivity Pattern of Different Antibiotics on Clinical Isolates of Escherichia coli Collected from Dhaka City, Bangladesh
}

\section{${ }^{1 *}$ SHAHRIAR, MOHAMMAD; ${ }^{1}$ HOSSAIN, MAHBOOB; ${ }^{2}$ KABIR, SHAILA}

\author{
${ }^{1}$ Department of Pharmacy, University of Asia Pacific, Dhanmondi, House no. 73, Road no. 5A, Dhanmondi, Dhaka-1209, Bangladesh \\ E-mail: shahriar_12@yahoo.com
}

${ }^{2}$ Faculty of Pharmacy, University of Dhaka, Dhaka-1000, Bangladesh

\begin{abstract}
A study of antimicrobial sensitivity of Escherichia coli isolated from clinical sources of Medinova Diagnostic Center and Popular Diagnostic Center, Dhaka, Bangladesh was carried out to facilitate the preference of drug in the management of Escherichia coli induced symptoms. Very low sensitivity of $E$. coli towards ampicillin (4\%), aztreonam (4\%), cloxacillin (5\%), nalidixic acid (5\%), ciprofloxacin $(7.5 \%)$, ceftriaxone $(12.5 \%)$, doxycycline $(12.5 \%)$, ceftazidime $(16.25 \%)$, co-trimoxazole (20\%), chloramphenicol $(22.51 \%)$, tetracycline $(25 \%)$, and netilmicin $(35 \%)$ was observed. Higher sensitivity pattern was observed for gentamicin (56\%) and only imipenem (95\%) has shown sensitivity pattern possibly susceptible enough to consider for the management of $E$. coli induced cases in the area under study. The low sensitivity to different antimicrobial could be attributed to their prevailing usage and abuse in the area under study. @ JASEM
\end{abstract}

Antimicrobial agents (antibiotics and related medicinal drugs) have substantially reduced the threat posed by infectious diseases. The use of these "wonder drugs", combined with improvements in sanitation, housing, and nutrition, and the advent of wide-spread immunization programmes, has led to a dramatic drop in deaths from diseases that were previously widespread, untreatable, and frequently fatal. These gains are now seriously jeopardized by another recent development: the emergence and spread of microbes that are resistant to cheap and effective first-choice, or "first-line" drugs. The bacterial infections which contribute most to human disease are also those in which emerging and microbial resistance is most evident. The consequences are severe. Infections caused by resistant microbes fail to respond to treatment, resulting in prolonged illness and greater risk of death. Treatment failures also lead to longer periods of infectivity, which increase the numbers of infected people moving in the community and thus ex-pose the general population to the risk of contracting a resistant strain of infection. Much evidence supports the view that the total consumption of antimicrobials is the critical factor in selecting resistance. E. coli is the most common cause of food and water-borne human diarrhea worldwide in developing countries causing 800000 deaths out of 650 million cases per year primarily in children under the age of five years (Turner et al., 2006). It also causes urinary tract infection and other complications in human. Depending on the virulence factors $E$. coli causes either non-inflammatory diarrhea or inflammatory diarrhea (Fratamico et al., 2004). Broad-spectrum antibiotics are used in chronic or life threatening cases (Doyle et al., 1993). In this study, the susceptibility of E. coli strains isolated in Dhaka city, Bangladesh was investigated against different antimicrobial agents to provide supportive implications for the proper treatment of E. coli induced infections and related complications.

\section{MATERIALS AND METHODS}

Patients were referred by community practitioners, clinics, and hospitals throughout Dhaka City, Bangladesh, to the Medinova and Popular Diagnostic Centre, private laboratory and clinical diagnostic facility, for evaluation of possible serious bacterial infection. Eighty isolates of $E$. coli from these patients were chosen for this study.

The specimens obtained were urine samples, pus samples, stool samples, blood samples. The specimens were collected according to Cheesbrough (1984). Specimens were cultured on XLD (Xylose lysine deoxycholate agar) and MacConkey agar plates, after which the cultural and morphological characteristics of the isolates were studied. Identification of isolates was done by standard microbiological methods as described by Cheesbrough (1984) and Cowan (1993). The antimicrobial sensitivity test of each isolate was carried out by the Kirby-Bauser disc diffusion method (Bauser et al., 1966) as per recommendation of National Committee for Clinical Laboratory Standards (NCCLS, 1997). This method allowed for rapid determination of invitro efficacy of a drug by measuring the diameter of the zone of inhibition that results from diffusion of the agent into the medium surrounding the disc. Mueller-Hinton agar plates were used for the disc diffusion tests. The discs used contained following antibiotics: ampicillin, AMP (25mcg); aztreonam, ATM (30mcg); ceftazidime, CAZ (30mcg); ceftriaxone, CRO (30mcg); chloramphenicol, CHL (30mcg); ciprofloxacin, CIP (5mcg); cloxacillin, CXL (5mcg); co-trimoxazole, CRX (25mcg); doxycycline, DOX (30mcg); 
A Survey on Antimicrobial Sensitivity Pattern.....

gentamicin, GEN (10mcg); imipenem, IPM(10mcg); nalidixic acid, NAL (30mcg) ; netilmicin, NET $(10 \mathrm{mcg})$ and tetracycline, TET $(30 \mathrm{mcg})$.

\section{RESULTS AND DISCUSSION}

Eighty clinical isolates of E. coli obtained from Medinova and Popular Diagnostic Centre, were subjected to antimicrobial sensitivity test against ampicillin, aztreonam, ceftazidime, ceftriaxone, chloramphenicol, ciprofloxacin, cloxacillin, cotrimoxazole, doxycycline, gentamicin, imipenem, nalidixic acid, netilmicin and tetracycline and the observed sensitivity was recorded (Table 1). With the sensitivity of $95 \%$, imipenem was demonstrated as the most susceptible antimicrobial followed by gentamicin $(56 \%)$. Incredibly diminutive sensitivity was observed for ampicillin (4\%), aztreonam (4\%), cloxacillin (5\%), nalidixic acid (5\%), ciprofloxacin $(7.5 \%)$, ceftriaxone $(12.5 \%)$, doxycycline $(12.5 \%)$, ceftazidime (16.25\%), co-trimoxazole (20\%), chloramphenicol $(22.51 \%)$, tetracycline $(25 \%)$, and netilmicin $(35 \%)$.

Table 1. Antimicrobial sensitivity pattern of clinical isolates of E. coli

\begin{tabular}{|c|c|c|c|c|c|c|c|c|}
\hline \multirow{2}{*}{ Isolates } & \multirow{2}{*}{$\begin{array}{l}\text { No. of } \\
\text { isolates }\end{array}$} & AMP & ATM & CAZ & CRO & CHL & CIP & $\mathrm{CXL}$ \\
\hline & & $3(4 \%)$ & $3(4 \%)$ & $13(16.25 \%)$ & $10(12.5 \%)$ & $18(22.5 \%)$ & $6(7.5 \%)$ & $4(5 \%)$ \\
\hline \multirow{2}{*}{ E. coli } & \multirow[b]{2}{*}{80} & CRX & DOX & GEN & IPM & NAL & NET & TET \\
\hline & & $16(20 \%)$ & $10(12.5 \%)$ & $45(56 \%)$ & $76(95 \%)$ & $4(5 \%)$ & $28(35 \%)$ & $20(25 \%)$ \\
\hline
\end{tabular}

Key: ampicillin = AMP; aztreonam = ATM; ceftazidime = CAZ; ceftriaxone = CRO; chloramphenicol = CHL; ciprofloxacin = CIP; cloxacillin = CXL; co-trimoxazole = CRX; doxycycline = DOX; gentamicin = GEN; imipenem = IPM; nalidixic acid = NAL; netilmicin = NET; tetracycline $=$ TET .

The variation found in the sensitivity pattern to these commonly used drugs in present study could be attributed to the prevailing usage and abuse of the drugs in the area under study. The lower sensitivity to the commonly used drugs indicates the dependence of the prescribers on these drugs in contrast to imipenem, which is less commonly used. This further suggests the relation between antibiotic usage and the level of drug resistance encountered. The judicious use of antibiotic by the health professional and efforts to control procurement and use of antibiotics officially in the locality will probably help to limit the increasing rate of drug resistance in the pathogens. Rational drug policy should be in use before potent antibiotics are introduced to the country (Aseffa and Yohhannes, 1996). Antibiotic administration should follow certain minimal requirements (Wellington and Van elsas, 1992). To restore efficacy, to earlier antibiotics and to maintain the success of new antibiotics that are introduced, it need to use antibiotics in a way, which assures an ecological balance that favors the predominance of susceptible bacterial flora (Bower, 1999). In Bangladesh, empirical therapy is the rule rather than the exception (Bennish, 1987) and in this context of changing the dynamics of resistance to antibiotics, it is imperative for optimal patient care that constant evaluation of antibiotic sensitivity pattern of pathogens for commonly used antimicrobial agents in a particular environment is carried out.

\section{REFERENCES}

Aseffa, A; Yohhannes, G. (1996). Antibiotic resistance pattern of Acinetobacter Sp. Journal of Infect Dis. 15:43-48
Bauser, AW; Kirby, WM; Sheris, JC; Truck, M. (1966). Antibiotic susceptibility testing by a standardized single disc method. American Journal of Clinical Pathology. 145: 225-230.

Bennish, M. (1987). The Bangladesh drug policy. The next step: using good drugs "goodly." Bangladesh J. Child Health. 11: 63-72.

Bower, JR. (1999). Foodborne diseases: Shiga toxin producing E. coli (STEC). Pediatr Infect Dis J. 18: 909-10.

Cheesbrough, M. (1984). Medical Laboratory Manual for Tropical Countries (Vol. 2: Microbiology), Tropical Health Technology/Butter- worth and Co., Cambridgeshire/Kent.

Cowan, ST. (1993). Cowan and Steel's manual forthe identification of medical bacteria, Cambridge University Press, London.

Doyle, J; Evans, Jr; Dolores, G; Blanco, J; Blanco, M; Gonzalez, EA, et al. (1993). Serotypes and colonization factors of enterotoxigenic Escherichia coli isolated in various countries. Eup. J. Epidemiol. 9:489.

Fratamico; Solomon, MC; Ayers, LW. (2004). Ceftriaxoneresistant Salmonella infection acquired by a child from cattle. N. Engl. J Med. 17:1242-1249.

National Committee for Clinical Laboratory Standards. Methods for dilution antimicrobial susceptibility tests for bacteria that grow aerobically, $3^{\text {rd }}$ ed, approved standard. NCCLS, Pennsylvania. Document M7-A3 (1997).

Turner, SM; Scott-Tucker, A; Cooper, LM; Henderson, IR. (2006). Weapons of mass destruction: virulence factors of the global killer enterotoxigenic Escherichia coli. FEMS Microbiology Letters 263(1): 10-20.

Wellington, EMH and Van Elsas, JD. (1992). Genetic interactions among microorganisms in the natural environment. Pergamon Press, Oxford. 